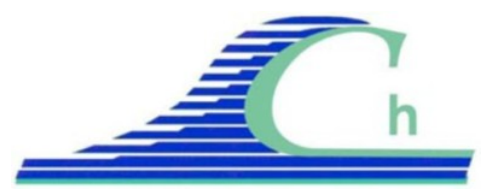

XII ${ }^{\text {èmes }}$ Journées Nationales Génie Côtier - Génie Civil

Cherbourg, 12-14 juin 2012

DOI:10.5150/jngcgc.2012.079-M @ Editions Paralia CFL

disponible en ligne - http://www.paralia.fr - available online

\title{
Utilisation des laitiers sidérurgiques dans les ouvrages portuaires et maritimes
}

\author{
Olivier MALASSINGNE ${ }^{1}$, Hervé COULON ${ }^{2}$, \\ Guirec PREVOT $^{3}$, Nicolas ROUXEL ${ }^{1}$
}

1. Centre d'Etudes Techniques de l'Equipement de l'Ouest, Département Laboratoire de Saint Brieuc, 5 rue Jules Vallès, 22000 Saint Brieuc, France.

\{olivier.malassingne,nicolas.rouxel\}@developpement-durable.gouv.fr ;

2. Centre d'Etudes Techniques de l'Equipement Nord-Picardie, Département Risques et Territoires, 42 bis rue marais, 59482 Sequedin, France.

herve.coulon@developpement-durable.gouv.fr

3. Centre d'Etudes Techniques Maritimes Et Fluviales,

2 boulevard Gambetta, BP 60039, 60321 Compiègne Cedex, France.

guirec.prevot@developpement-durable.gouv.fr

\section{Résumé :}

Le terme générique de laitier sidérurgique englobe plusieurs matériaux aux caractéristiques physico-chimiques différentes. Ils sont cependant élaborés en véritables co-produits de l'acier et de la fonte. Leur utilisation ultérieure est intégrée dans les process industriels de fabrication.

Les laitiers d'aciérie de conversion comme de fours électriques peuvent être utilisés en ouvrages maritimes sous forme de granulats, enrochements ou matériaux tout venant de quai. Ils présentent les caractéristiques mécaniques générales équivalentes à celles des granulats naturels et obéissent aux mêmes normes et recommandations d'usage. Quelques points spécifiques liés au mode d'élaboration des laitiers d'aciéries nécessitent néanmoins quelques précautions complémentaires (maîtrise du potentiel de gonflement lié à la présence de chaux libre et vérification des teneurs en métaux lourds).

Mots-clés : Travaux maritimes - Laitiers de Hauts Fourneaux et d'aciéries - Scories

\section{Introduction}

Comme tout processus industriel, la production de fonte et d'acier s'accompagne de la formation de co-produits dont l'essentiel constitue les laitiers. Historiquement, les sidérurgistes se débarrassaient de ces matériaux en les évacuant dans des zones de stockage (crassiers). L'émergence de filières de valorisation se sont organisées dans les années 1930, notamment suite à l'invention du Tarmacadam (intégration de granulats de laitiers dans du goudron). L'industrie cimentière s'est ensuite largement impliquée dans la valorisation des laitiers de haut-fourneau comme substitut du clinker avec un coût de production nettement plus faible. Sur les trois principaux centres sidérurgiques de France deux sont situés en zone portuaire, Fos-sur-Mer (13) et Dunkerque (59). 


\section{Les différents types de laitiers}

L'industrie métallurgique est basée sur deux filières principales qui génèrent toutes deux des laitiers sidérurgiques.

- Filière fonte : Cette filière produit de l'acier à partir du minerai de fer fondu en hautfourneau pour élaborer la fonte. Cette fonte liquide est ensuite transférée en installation de conversion (aciérie avec convertisseur à oxygène) pour produire l'acier.

- Filière ferrailles : Cette seconde filière est basée sur le recyclage des métaux qui, après récupération, sont refondus dans des aciéries où l'énergie de fonte est fournie par arc électrique. Ce processus produit des laitiers spécifiques dénommés laitiers d'aciérie de four électrique (LAFE). Ils se subdivisent en "filière carbone" et "filière inox" selon la nature des ferrailles enfournées et la nuance de l'acier voulue.

Le recensement exhaustif réalisé par le CTPL (site web) en 2008 établit la production annuelle française à environ 5,7 millions de tonnes toutes filières confondues. Cette production a ensuite été fortement impactée par la crise financière. Les laitiers de hautfourneau (LHF) sont pratiquement tous valorisés dans l'industrie cimentière et les techniques routières. Il n'existe pratiquement pas de stocks de LHF. En revanche les stocks historiques des laitiers d'aciéries restent très importants et continuent à être alimentés par la production annuelle. Environ $20 \%$ de la production annuelle de laitiers d'aciéries ne trouve pas de débouchés en valorisation.

Depuis 2008, c’est la Directive Cadre Déchets n 2008/98/CE, traduite en droit français par l'Ordonnance $\mathrm{N}^{\circ}$ 2010-1579 du 17 décembre 2010, qui fixe les exigences et objectifs auxquels les Etats Membres de l’Union Européenne doivent se conformer. Cette Directive européenne ouvre la possibilité pour certains déchets, sous certaines conditions, de changer de statut juridique. A ce jour, les laitiers conservent le statut de déchet industriel selon le code de l'environnement (Livre V - Titre IV). Ils entrent dans la codification suivante : déchets non dangereux issus de procédés thermiques. Le principal enjeu du règlement REACH auquel ils sont soumis, est de combler le déficit de connaissances des risques environnementaux et sanitaires qui peuvent résulter de la production et de l'utilisation des substances chimiques en imposant aux producteurs de substances de prouver que les risques liés à ces substances sont valablement maîtrisés (REYNARD, 2009).Malgré leur statut juridique, certains laitiers sont utilisés depuis très longtemps en techniques routières et en industrie cimentière. Ils sont donc encadrés par un référentiel normatif et réglementaire qui s'applique à ces usages.

\section{Laitiers de haut-fourneau (LHF)}

La première étape dans la production d'acier est l'obtention de fonte, par fusion du minerai de fer grâce à la combustion du coke dans un haut fourneau. Le fer fondu (fonte) et le laitier, sont sous-tirés en fusion du haut-fourneau. Chaque tonne de fonte génère de l'ordre de $300 \mathrm{~kg}$ de laitier. Selon le processus de refroidissement du laitier en fusion, on distingue deux familles : 


\section{XII ${ }^{\text {èmes }}$ Journées Nationales Génie Côtier - Génie Civil \\ Cherbourg, 12-14 juin 2012}

- LHF cristallisé : Il est obtenu par refroidissement lent à l'air qui permet sa cristallisation sous forme de roche dure artificielle. Le laitier est ensuite arrosé afin d'abaisser sa température et de le fragmenter grossièrement par choc thermique. A la fin de la cristallisation le matériau est ensuite concassé et criblé pour obtenir des granulats calibrés.

- LHF vitrifié : Le laitier en fusion est arrosé abondamment sous haute pression pour le vitrifier. Le choc thermique provoque son explosion et forme instantanément du laitier vitrifié, appelé laitier granulé.

\subsection{Caractéristiques environnementales et maîtrise de la qualité}

Il n'existe pas d'exigences particulières relatives à la caractérisation environnementale préalable à la valorisation des LHF. Le programme européen SESAR (StEel Slag roAd and enviRonment) s'est intéressé à l'identification des éléments potentiellement lixiviables et à leurs mécanismes de relargage. Cette expérimentation a mis en évidence certains couplages physico-chimiques et en particulier les corrélations existantes entre conditions de $\mathrm{pH}$ et solubilisation des sulfates et ions calcium pour les LHF.

La composition du laitier est contrôlée, de façon régulière et par analyse chimique, lors de son élaboration ou déduite des différents éléments enfournés.

\subsection{Filières de valorisation actuelles}

En raison de leurs caractéristiques granulométriques et mécaniques les laitiers de hauts fourneaux peuvent être assimilés à des matériaux de classes GTR D21 ou D31 selon leurs coupures granulométriques. Ils sont donc utilisables en remblais et couches de formes non traitées selon les règles définies dans le Guide Technique Réalisation des remblais et couches de forme (SETRA-LCPC, 1992). Néanmoins, même si ces usages ont été très développés par le passé, les propriétés particulières de ces matériaux et leur raréfaction les réservent à des usages plus nobles. Le laitier concassé est utilisé plus fréquemment comme granulat des structures de chaussées dans les graves traitées aux liants hydrauliques, quel que soit le liant utilisé. Certains granulats de laitier cristallisé concassé, en raison de leurs propriétés mécaniques mesurées, conviennent pour la fabrication des enrobés hydrocarbonés, y compris pour la réalisation de couches de surface.

En technique routière, le laitier vitrifié est utilisé actuellement comme liant pour la réalisation d'assises de chaussées sous la forme de graves traitées ou de sables traités mais également en traitement de sols. Ils sont obtenus par mélanges et broyage de coproduits de l'industrie, d'activants de type chaux, cendres volantes voire de clinker.

Les laitiers vitrifiés constituent un composant important de l'industrie cimentière et permettent de réaliser de nombreux ciments normalisés de construction. Ils sont principalement utilisés dans la production de ciment, en remplacement du clinker, dans les catégories CEM II et CEM III (jusqu’à 95 \% du clinker) destinées notamment à la 
réalisation de bétons en milieux agressifs (alcalin ou marin) où sa présence favorise la résistance à l'alcali-réaction et protège les armatures (CTPL, site web).

A ce jour, en raison de la crise locale de la sidérurgie entraînant une raréfaction de la ressource, la grande majorité du laitier est valorisée dans sa forme vitrifiée au profit de l'industrie cimentière. Cette prédominance importante rend difficile l'émergence de nouvelles filières de valorisation par manque de ressource du sous-produit.

\section{Laitiers d'aciérie de conversion (LAC)}

Le laitier de convertisseur à oxygène est issu de la fabrication d'acier à partir de la fonte liquide obtenue en haut-fourneau. Cette dernière est affinée (soufflage d'oxygène) par oxydation du carbone dans un convertisseur d'où sort l'acier. Des ajouts de chaux sont nécessaires pour protéger le convertisseur et entrent dans les multiples réactions chimiques d'oxydation qui contribuent à la décarburation de la fonte.

En fonction du point d'injection d'oxygène dans le convertisseur, on distingue :

- le laitier LD (Linz-Donowitz) où l'oxygène est insufflé par le haut,

- le laitier LWS (Loire-Wendel-Sprunck) où l'oxygène est insufflé par le fond.

On parle couramment des laitiers LD (ou scories LD) recouvrant en fait sous ce terme générique les laitiers LD et LWS. Chaque tonne d'acier produite génère de l'ordre de $100 \mathrm{~kg}$ de laitier (CTPL, site web).

\subsection{Caractéristiques environnementales et maîtrise de la qualité}

Tout comme pour les LHF, des études sur le potentiel polluant ont été menées au niveau européen (programme SESAR). Le potentiel expérimental de relargage des ions $\mathrm{Cu}$ et pour moindre part des ions Cr est avéré en fonction du potentiel électrochimique des solutions de lixiviation. Classiquement, la qualité du laitier d'aciérie de conversion est contrôlée, de façon régulière. La maîtrise de ces procédures a permis à Arcelor Mittal de proposer sur le marché un Liant Hydraulique Routier (LHR) à base de laitiers LD agréé. Pour les stocks de "génération ancienne" qui sont généralement qualifiés de "scories LD", qui ne relèvent pas de la maîtrise des paramètres de production, les mêmes principes de qualification mécanique, environnementale et de traçabilité sont appliqués. En revanche leur valorisation se limite à des usages classiques (matériaux non revêtus permettant de négliger le potentiel de gonflement).

\subsection{Contexte normatif particulier}

En complément des normes relatives à l'ensemble des laitiers, il existe une norme de référence, spécifique aux laitiers d'aciérie (NF EN 1367-3). Actuellement, la mesure du potentiel de gonflement est réalisée par l'essai de gonflement accéléré à la vapeur (NF EN 1744-1). 


\section{XII ${ }^{\text {èmes }}$ Journées Nationales Génie Côtier - Génie Civil \\ Cherbourg, 12-14 juin 2012}

\subsection{Filières de valorisation actuelles}

Toutes filières confondues, les laitiers d'aciéries sont valorisés comme matériau de viabilité dans les techniques de génie civil comme les remblais et la protection des berges. Leur masse volumique plus importante que celle d'un granulat naturel permet une utilisation en masque ou en éperons drainants (OFRIR LCPC, 2002). Les domaines d'utilisation des laitiers LAC sont les domaines où les exigences de stabilité volumique du matériau ne sont pas essentielles. L'utilisation se limite à des applications de graves non traitées (GNT), à des remblais généraux, matériaux drainants, assainissement, pieux ballastés, renforcement de berges, enrochements (OFRIR LCPC, 2002) où sa densité élevée permet de résister au phénomène d'affouillement (CTPL, site web).

Une autre utilisation possible de ces laitiers se situe dans les couches de roulement en raison de leurs qualités mécaniques très performantes. Mais les premières expériences réalisées (dans les années 70) ont conduit à des désordres après quelques mois (excroissances à la surface du revêtement). Des études ont montré que ces problèmes pouvaient être sensiblement limités par un concassage suivi d'un vieillissement du laitier (vieillissement à l'air, à l'eau, à l'eau chaude) (RAYSSAC et al., 2009). De la même façon, les laitiers LAC pourraient éventuellement être utilisés pour la réalisation d'enduits superficiels après un vieillissement par simple stockage à l'air libre pendant 6 mois (OFRIR LCPC, 2002).Sa richesse en chaux, permettant un phénomène de prise sans traitement particulier, est souvent mise à profit dans la réalisation de couches de roulement "rustiques" et très économiques pour des pistes de chemins non revêtus. Les laitiers de nouvelle génération dont le processus de contrôle en continu des caractéristiques géochimiques est maîtrisé entrent dans la composition de liants LHR.

Toujours en raison de la densité élevée des laitiers LAC, des essais de bétons spéciaux ont été réalisés dans les années 1980 dans le Calaisis pour la réalisation de quelques accropodes de défense littorale. Ces ouvrages expérimentaux qui présentaient des caractéristiques très adaptées après leur mise en œuvre ont très mal vieilli en subissant des phénomènes de gonflement différés qui ont très fortement endommagé les ouvrages.

\subsection{Recommandations pour l'utilisation des laitiers d'aciérie bruts en milieu maritime}

Arcelor Mittal a élaboré une fiche à l'usage des maîtres d'ouvrage et des maîtres d'œuvre (ARCELOR MITTAL, 2007). Les principales recommandations sont les suivantes pour un produit conforme aux réglementations en vigueur :

Pour toute utilisation en milieu aquatique, il convient de s'assurer que les débits d'eau permettent à tout moment une dilution suffisante pour éviter toute augmentation sensible du $\mathrm{pH}$ du milieu. Cette augmentation sera d'autant plus importante que le laitier sera fin et riche en chaux libre et que le débit d'eau sera faible.

Pour ce qui concerne l'immersion en milieu marin, des travaux réalisés ont montré que les échanges avec l'eau de mer se sont traduits par une légère augmentation du pH à proximité immédiate de la zone d’immersion, voir figure 1. 
Les effets sur les autres éléments mesurés ont été très réduits, voire inexistants ou non imputables au produit immergé. Les préconisations d'utilisation sont, en conséquence, les suivantes :

- Pour les utilisations au large ou dans les bassins à marée, le produit peut être utilisé sans restriction sur le plan environnemental. Compte tenu de sa granulométrie et de sa densité, la dispersion de la matière est très réduite.

- Pour les utilisations en bassin portuaire semi-ouverts (bassins à flot), il y a lieu de vérifier que le ratio $\mathrm{L} / \mathrm{S}=$ =rapport liquide/solide, exprimé en $\mathrm{m}^{3}$ d'eau par tonne de laitier immergé soit au moins de 100. Il n’est pas nécessaire de protéger systématiquement la partie émergée. En effet, après quelques temps, une prise par hydratation de la chaux s'instaure et le talus devient résistant à la houle danss les bassins. Les pentes des talus sont raides et sont identiques sous l'eau et hors d'eau, voir figure 2.

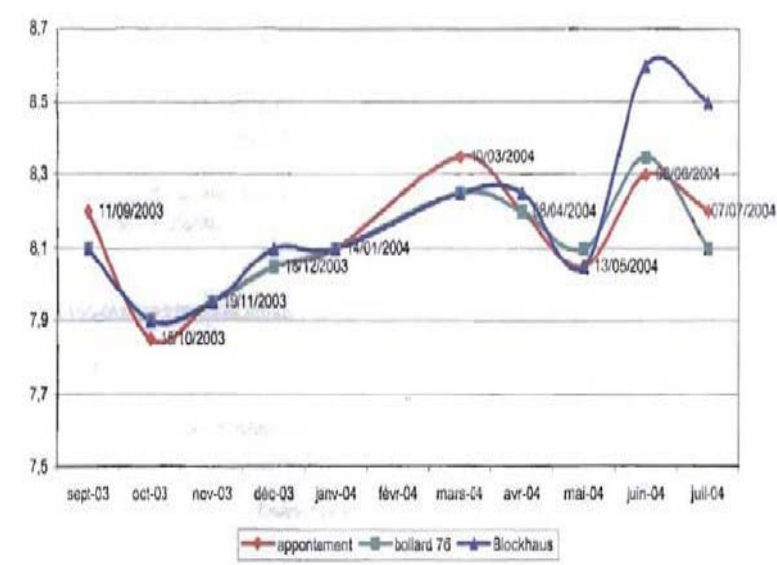

Figure 1. Évolution du $\mathrm{pH}$ de l'eau de mer entre septembre 2003 et juillet 2004, Port de Dunkerque Darse de l'appontement céréalier.

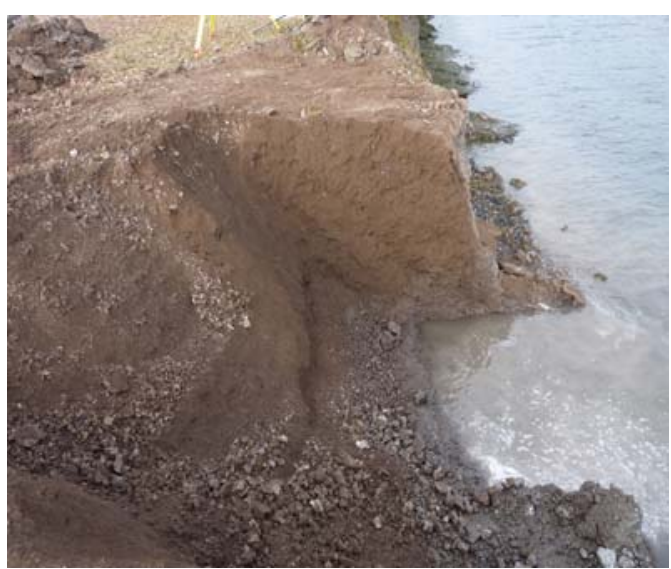

Figure 2. Sondage à la pelle mécanique mettant en évidence la paroi sub-verticale en front de darse (COULON, 2011). Port de Dunkerque

Darse de l'appontement céréalier.

Les surfaces remblayées peuvent être utilisées à des fins d'aires de stockage. La partie émergée du laitier d’aciérie ne doit pas être recouverte par un revêtement étanche. L'expansion volumique de la chaux libre causerait au revêtement des dégâts sensibles. Tout au plus, une émulsion bitumineuse légère peut être mise en œuvre afin de limiter la production et l'envol de poussières sur les surfaces soumises à circulation routière. Aucun bâtiment ni fondation ne doivent être installés sur un remblai en laitier d'aciérie, même s’il est recouvert par un autre matériau. Bien que les impacts sur la qualité de l'eau soient réduits à une légère variation du $\mathrm{pH}$, il est recommandé de ne pas autoriser la baignade à proximité du lieu du remblai, durant la période de mise en œuvre des matières. Un suivi par réalisation d'analyse sur les sédiments du bassin et des matériaux 


\section{XII ${ }^{\text {èmes }}$ Journées Nationales Génie Côtier - Génie Civil \\ Cherbourg, 12-14 juin 2012}

mis en œuvre est nécessaire en vue de connaître et de maîtriser l'impact sur l'environnement.

\section{Laitiers d'aciérie de four électrique (LAFE)}

Les laitiers d'aciéries électriques proviennent de la production d'acier par fusion des ferrailles recyclées dans des fours électriques. Ils sont issus de deux procédés utilisant des fours électriques soit pour la production d'aciers faiblement alliés, dits "carbone", soit pour la production d'aciers inoxydables (inox). L'élaboration des aciers dits "carbone" se fait généralement en deux étapes, l'une au four de fusion et l'autre en poche de coulée, ce qui génère deux types de laitiers. Celle de l'acier inoxydable comporte une troisième étape intermédiaire d'oxydation du carbone formant le laitier AOD (Argon Oxygen Decarburation). Pour les deux filières se sont essentiellement les laitiers de four qui sont valorisés. Les laitiers de poche, en raison de leur forte basicité (très riches en chaux) sont soit recyclés directement au niveau des aciéries, soit en filières d'amendement agricole sous réserve de la maîtrise des polluants éventuels.

\subsection{Caractéristiques environnementales}

Les résultats de planches expérimentales publiées par le CTPL (CTPL, site web) indiquent des taux de relargages des principaux métaux lourds très faibles. Néanmoins, ils sont sensiblement plus élevés en chrome pour les laitiers de four de la filière inox.

\subsection{Filières de valorisation actuelles}

Toutes filières confondues, le laitier d'aciérie est valorisé comme matériau de viabilité dans les techniques de génie civil comme les remblais et les couches de forme. Leur masse volumique plus importante que celle d'un granulat naturel permet une utilisation en masque ou en éperons drainants (OFRIR LCPC, 2002).

La réponse favorable vis-à-vis des tests de gonflements et la très faible teneur en chaux libre du laitier d'aciérie de four électrique devraient permettre son exploitation dans le cadre de la fabrication de "Graves traitées aux liants hydrauliques".

Compte tenu de ses caractéristiques géomécaniques intrinsèques très intéressantes, les laitiers d'aciérie de four électrique peuvent être exploités en association avec un liant bitumeux. Il est également un matériau d'ajout pour améliorer le Coefficient de Polissage Accéléré de certains granulats naturels (OFRIR LCPC, 2002) propriété particulièrement recherchée lorsque des surfaces anti-glissement sont nécessaires. En raison de leur densité élevée, ces matériaux peuvent être utilisés en protection ou confortement de berges si leur innocuité vis-à-vis de l'environnement est démontrée.

\section{Conclusion}

Le terme générique de laitier sidérurgique englobe plusieurs matériaux aux caractéristiques physico-chimiques différentes. Ils sont cependant élaborés en véritables 
co-produits de l'acier et de la fonte. Leur utilisation ultérieure est intégrée dans les processus industriels de fabrication. Les laitiers de hauts fourneaux ont depuis longtemps fait l'objet d'une réutilisation grâce à leurs performances. La quasi-totalité de la valorisation actuelle est effectuée par l'industrie cimentière comme liants routiers ou adjuvants de ciments. Les laitiers d'aciérie de conversion comme de four électriques peuvent être utilisés en ouvrages maritimes sous forme de granulats, enrochements ou matériaux tout venant de quai. Ils présentent les caractéristiques mécaniques générales équivalentes à celles des granulats naturels et obéissent aux mêmes normes et recommandations d'usage. Quelques points spécifiques liés au mode d'élaboration des laitiers d'aciéries nécessitent néanmoins quelques précautions complémentaires conditionnées par la maîtrise de leur potentiel de gonflement lié à la présence plus ou moins importante de chaux libre et de magnésie.

\section{Références bibliographiques}

ARCELOR MITTAL (2007) Utilisation de laitiers d'aciérie bruts en milieu maritime [URL http://www.ctpl.info/documents/ldmer.pdf ].

COULON H. (2011). Filières de valorisation des déchets du BTP et des co-produits industriels de la région Nord-Pas de Calais. Guide d'aide à la décision à destination des M d'Ouvrage, Maîtres d'Oeuvre et Bureaux de Contrôles. CRGV, CETE Nord Picardie. [URL http://www.nord.equipement-agriculture.gouv.fr/IMG/pdf/Guide_regional_dechets_et_coproduits_juin_2011_cle24d2b5.pdf ]

CTPL (site web). http://www.ctpl.info/

OFRIR LCPC (2002). http://ofrir.lcpc.fr/accueil/accueil_articles_theme.php

RAYSSAC E., AURIOL J.C., DENEELE D., DE LARRARD F., LEDEE V., PLATRET G. (2009). Valorisation de laitiers d'aciérie LD pour les infrastructures routières. Bulletin de Liaison des Laboratoires des Ponts et Chaussées, avril-mai-juin, n²75, pp 27-38.

REYNARD J. (2009). Les laitiers sidérurgiques et le règlement REACH. Revue générales des routes, février, n873, pp 45-47.

SETRA, LCPC (1992). Guide technique de réalisation des remblais et des couches de forme. 